\title{
Algorithm for Design of Digital Notch Filter Using Simulation
}

\author{
Dr. Amit Verma \\ Professor, Chandigarh University, \\ Ghauran
}

\author{
Naina \\ Electronics \& Communication \\ Engineering \\ Ambedkar Institute of Advance \\ Communication Technologies \\ \&Research (A.I.A.C.T\&R) \\ Delhi,India
}

\author{
Mrs. C.S. Vinitha \\ Electronics \& Communication \\ Engineering \\ Ambedkar Institute of Advance \\ Communication \\ Technologies\&Research \\ (A.I.A.C.T\&R) \\ Delhi,India
}

\begin{abstract}
- a smooth waveform is generated of low frequency signal can be achieved through the Digital Notch Filter. Noise can be easily eliminated from a speech signal by using a Notch filter. In this paper the design of notch filter using MATLAB has been designed and implemented. The performance and characteristics of the filter has been shown in the waveform in the conclusion part of the paper.
\end{abstract}

Keywords-Component; filters; design of filters; MATLAB etc

\section{INTRODCTION}

Signal Processing is the area of Engineering that deals with the processing of electrical signal either in discrete form or in continuous form. The signal need to be processed that the information that they contain can be converted, displayed and analyzed to other form that may be used as a part of Analog signal processing is a part of signal processing where the continuous -time signal are to processed to get the desired results.

The area that deals with the discrete time signal in digitized form is known as Digital Signal Processing. Here a digitized signal as a number sequences at discrete time. There are many reasons why the digital signal processing of an actual (naturally analog) signal may be preferable. Programmability features of digital signal processing technique is one of the most important merit the make it superior to its analog counterpart. Other important features are accuracy and precision.

The subject of Digital Signal Processing is very wide. It consists of study of various mathematical tools like FourierTransform, Z-Transform, DFT, FFT etc. In addition to sound understanding of the simulation tools such as: MATLAB .A very important area of Digital Signal Processing, where huge numbers of efforts is being invested is the area of realized filter. Efficient techniques and algorithms are being worked out and are widely available in literature in this paper we shall see that the realization of a digital notch filter and also see that the how removing noise from a speech signal by using GUI Model in MATLAB software and how the noise easily removed by using inverse filtering.
In this paper we shall know that how we can design a filter byusing a MATLAB software .There are many types of filters are available.

\section{Digital TeChNOLOGY}

First of all, we discuss about the Digital Technology that how significant is that in our life. 1) Digital signals can be represented by electronics $\mathrm{ON}-\mathrm{OFF}$ switches.2) coding helps maintaining secrecy in transmission.3) Data-compression techniques helps in effective economy in transmission.4) signal enhancement and signal reduction can be effected by introducing 1's and 0's appropriate places, as required.5)one of the major advantage of digital transmission of signal is its ability to reduce noise. In transmission of signal, noise gets added to the signal in the form of amplitude disturbances. Since, the binary signals have only two levels (1's and 0's) ofoperation; we can reduce noise by limiting the amplitude. 6) Modern VLSI and ULSI technologies have made circuit complexities a simple affair. They have introduced high - speed digital processor into the open market at affordable prizes. This has resulted in the popularity of DSP among common man.

\section{TYPES OF FILTERS}

Filters are the networks that process signal in frequency dependent manner. The basic concept of a filter can be explained by examining the frequency dependent nature of the Impedance of capacitors and inductors. There are main two types of Filters are:1) Analog Filters and 2) Digital Filters. The Analog Filters are like Low Pass Filter, High Pass Filters, Band Pass Filters and Band Reject Filters. And the Digital filters like Impulse Invariant Response (IIR) Filters and Finite Impulse Response (FIR) Filters.

\section{DESIGN OF DIGITAL FILTERS}

The procedure presented here for the design of digital filter is based on these some steps:

We first developed the expression for $\mathrm{H}(\mathrm{s})$ based on the analog filterdesign and then modify it in an appropriate fashion to suit the digital domain. 
As an example consider the expression for the $\mathrm{H}(\mathrm{s})$ of the second order analog filter:

$$
H(s)=\frac{\omega_{c}^{2}}{s^{2}+2 \delta \omega_{c}^{s}+\omega_{c}^{2}}
$$

Where $\omega c=$ cut of frequency and =damping factor. To get transfer function desired digital filter, we first take the inverse Laplace Transform of above equation to convert frequency domain expression of $\mathrm{H}(\mathrm{s})$ into its time domain equivalent $h(t)$.

After $\mathrm{h}(\mathrm{t})$ is obtained we used Z-Transform to convert it into $H(Z)$, we construct the required digital filter . This type of design is called "impulse invariant design".

We may also convert $\mathrm{H}(\mathrm{s})$ directly from s-domain into $\mathrm{H}(\mathrm{z})$ in z-domain by means of what is known as Bilinear Transformation. Once the $\mathrm{H}(\mathrm{z})$ is obtained, we proceed in the same way as in the case of impulse -invariant-design method to construct the desired digital filter.

\section{A. Design of Digital InfiniteImpulse Response (IIR) Filters} Filters.

There are several methods to designing the digital IIR

\section{1) BUTTERWORTH IIR FILTER}

Thedesign of Butterworth filters design with the design of its analog Counter Part from the analog design Step. We find expression for $\mathrm{H}(\mathrm{s})$. From $\mathrm{H}(\mathrm{s})$ we find $\mathrm{H}(\mathrm{z})$ either by direct conversion or with the help of a suitable transformation. Then using $\mathrm{H}(\mathrm{z})$ we can construct the desired filter structure.

Normalized the frequency-To normalize the frequency we use the equation:

$\omega=2 \pi f$

\section{2) CHEBYSHEV FILTER}

There are two types of Chebyshev IIR filters. They are type-I(regular) chebyshev filter and the type-II (inverse) chebyshev filters, which is characterized by pass band containing no ripples and stop band containing ripple.The chebyshev design makes use of chebyshev polynomials, given by:

$$
|H(\omega)| \text { in } d B=-10 \log \left(1+\varepsilon^{2} c_{n}^{2}\right)
$$

Where $\varepsilon=$ amount of ripple in the magnitude, and $=$

Coefficient $\mathrm{Cn}$ is given by:

$$
c_{n}=\cosh \left\{n \cosh ^{-1} \frac{\omega}{\omega_{c}}\right\}, \quad \text { if } \frac{\omega}{\omega_{c}} \geq 1
$$

\section{3) BILINEAR TRANSFORMATION}

THE Bilinear or Tustin Transformation IS A mathematical relation by which we can convert an s-domain equation directly into its equivalent in the $\mathrm{z}$-domian. The expression of the bilinear transformation is given by $\mathrm{S}=$ :

$$
\frac{2\left(1-z^{-1}\right)}{T\left(1+z^{-1}\right)}
$$

Where $T=$ sampling interval

\section{B. Design of Digital Finite Impulse Response (FIR) Filters}

There are many methods of designing FIR filter like window Techniques these techniques are like: rectangularwindow, Hamming window,Hanningwindow ,Blackman window ,Bartlett window and Kaiser window .

\section{PROPOSED ALGORITHM}

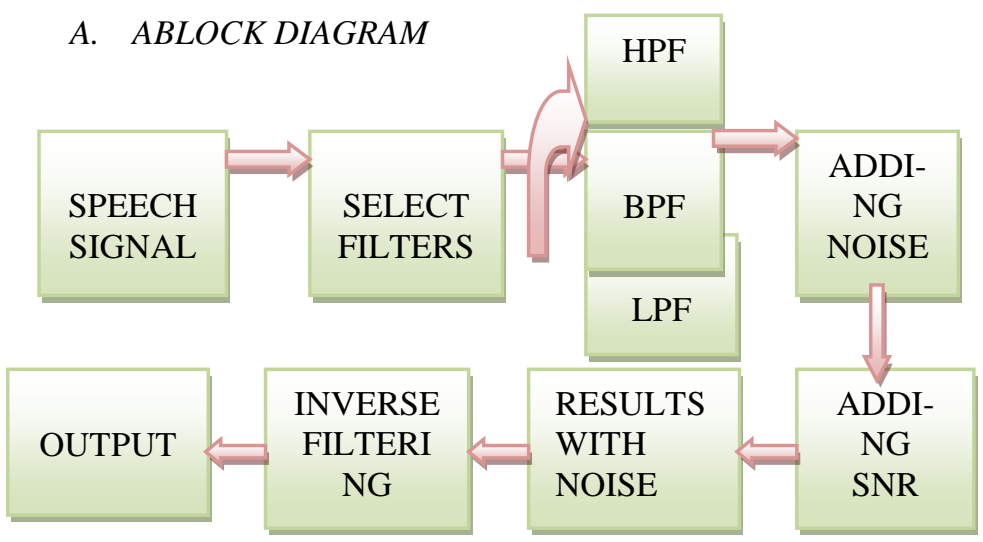

Above block diagram shows complete process of the system according to this block diagram this process is done in MATLAB software. First of all, we will take an input of speech signal or an audio signal.

Then we select a filter like low pass, high pass or band pass and select the frequency of the selected filter and proceeding further and can see the waveform and spectrum of a speech signal and after that we have to add the noise as per user choice and then we can see the result of a noisy signal which is not our output. For eliminating noise from the speech signal we have do the inverse filtering or denoising of the signal. After that we can get the eliminated noise speech signal. We can get a noise free speech signal only then when we denoising the signal or doing inverse filtering.

\section{B NOTCH FILTER}

The NOTCH FILTER is a filter that passes all the frequencies except those in a stop band centered on a centered frequency. A closely related knowledg item discusses the concept of the $\mathrm{Q}$ filter. The amplitude response of a notch filter is flat at all frequencies exept for the stop band on either side of the centered frequency. The standard reference point for the roll-off on each side of the stop band are the points where the amplitude decreased by $3 \mathrm{db}$ to $70.7 \%$ of its original amplitude. Many people think that the higher the $\quad$ Q then deeper the notch this is not true. The depth of a notch depends on the matching of components

\section{MATLAB SOFTWARE}

This process we are doing on MATLAB software. For eliminating the noise from our speech signal we are using GUI 
Tool in Matlab program. This is the mathematical tool which is used for all type of mathematical calculations or mathematical process. This software is used widely in these days and this software can be easily applicable on digital processing, image processing, signal processing, and control engineering many more fields.

\section{RESULTS}

\section{A. RESULTS WHEN WE UPLOAD A SPEECH SINPUT}

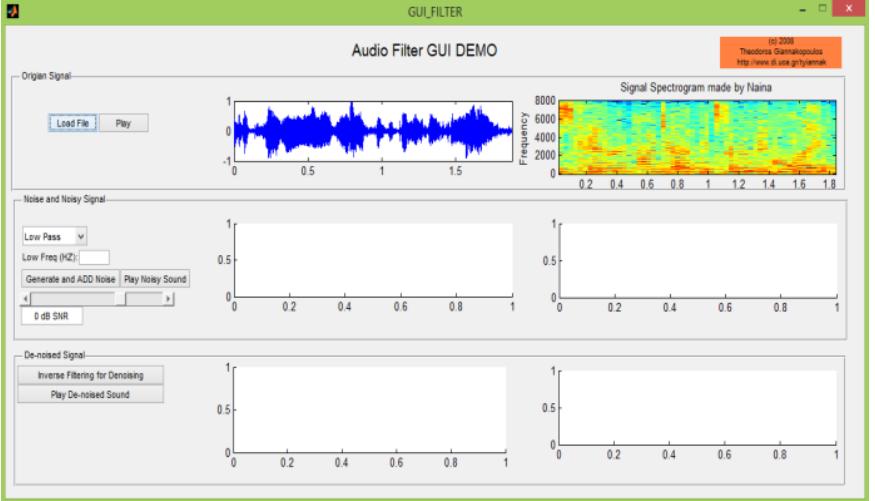

Fig.1. AUDIO FILTER GUI DEMO OF A SPEECH SIGNAL

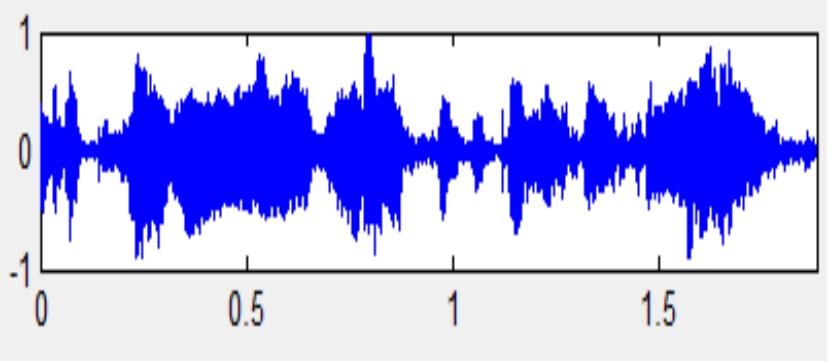

Fig.2. Waveform of original speech signal

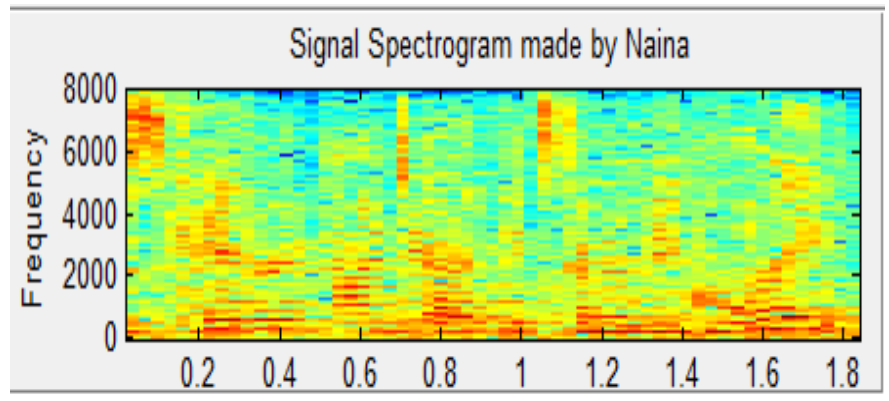

Fig.3. Spectrum of a speech signal

\section{B. RESULTS WHEN NOISE IS ADDED TO THE SIGNAL}

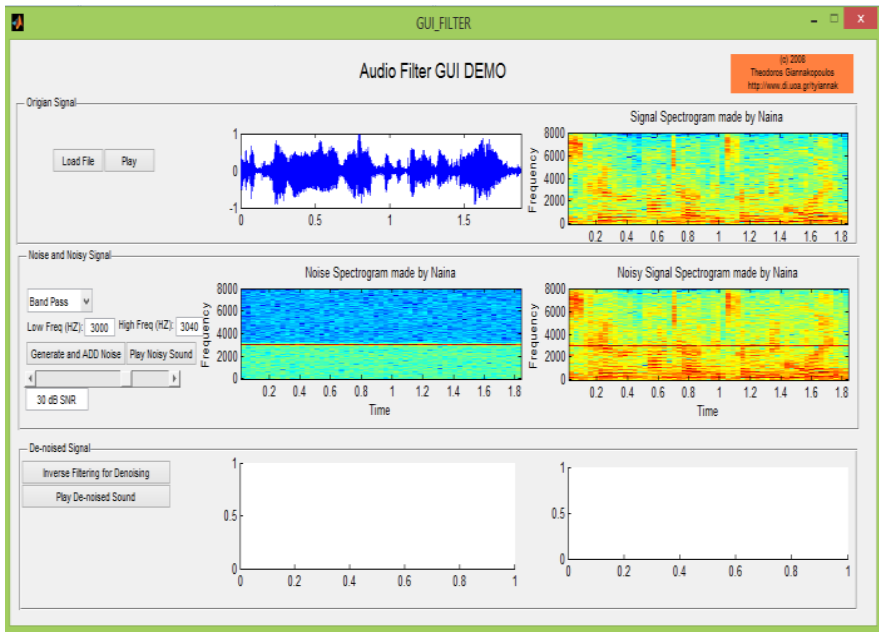

Fig.4. AUDIO FILTER GUI DEMO WHEN NOISE IS ADDED IN THE SPEECH SIGNAL

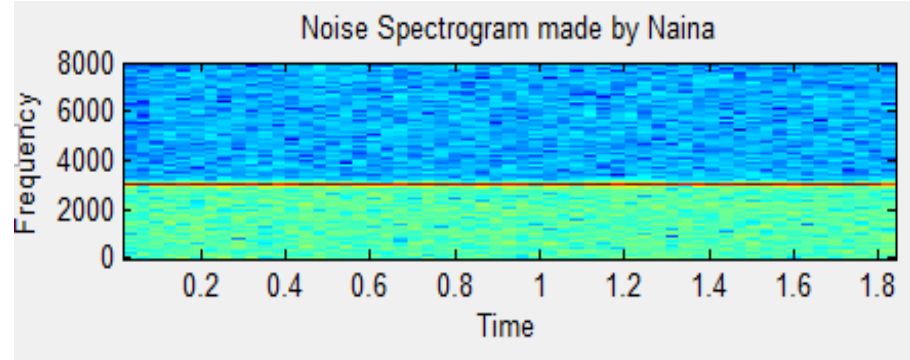

Fig.5. Waveform of the speech signal after adding the noise

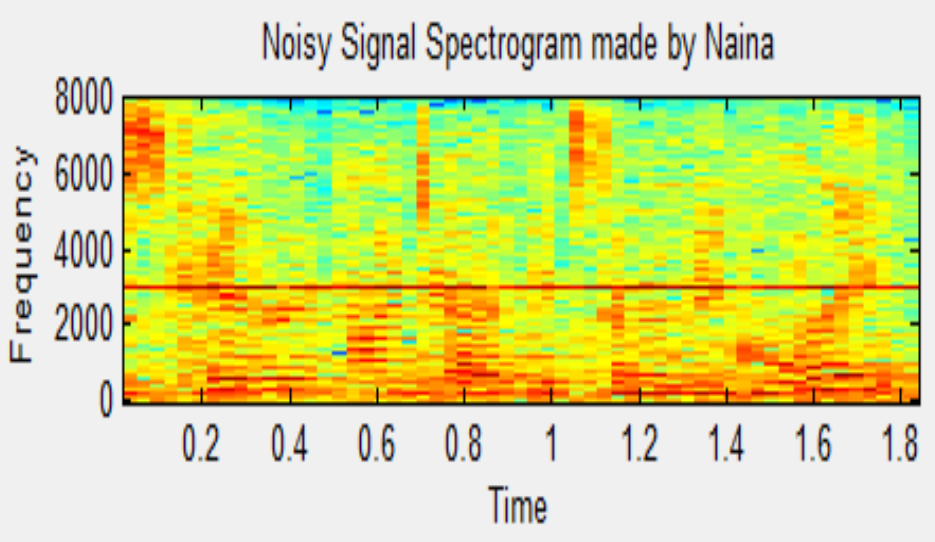

Fig.6. Spectrum of a signal when noise is added to the speech signal 


\section{RESULTS AFTER INVERSE FILTERING FOR DENOISING THE SPEECH SIGNAL}

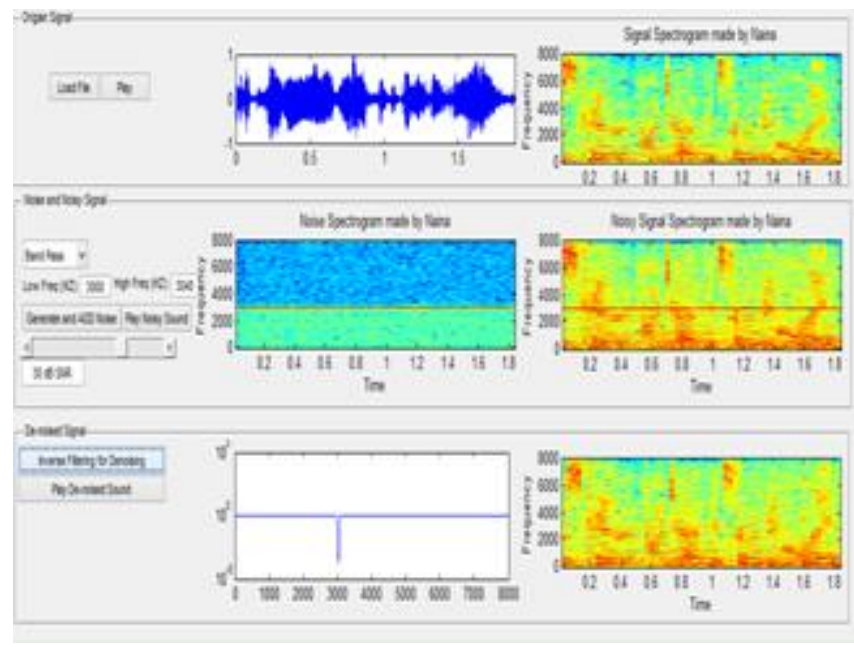

Fig.7. Audio filter GUI demo after inverse filtering for denoising the speech signal

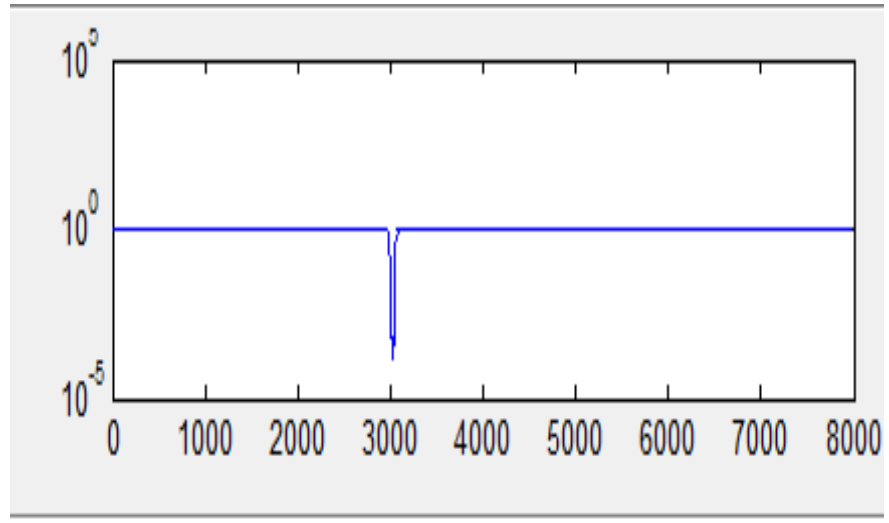

Fig.8. Noise eliminated by using the notch filter

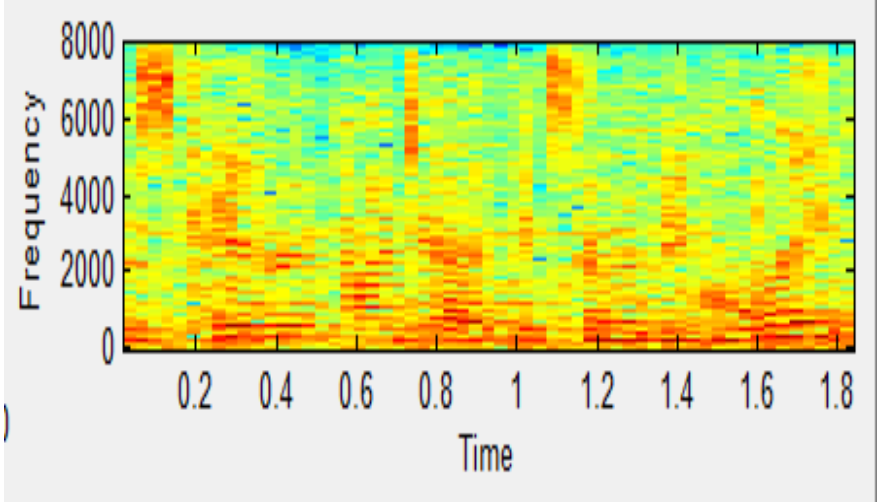

Fig.9. Spectrum of a notch filter after Denoising the speech signal

\section{CONCLUSION}

In this paper we have seen that how we can easily eliminated the noise from the speech signal or a random signal by using a GUI Tool in MATLAB software .and also seen the design of notch filter by doing inverse filtering. This technique can easily use in speech signal processing for eliminating the noise from the random or a speech signal. We can test this process that weather is it correct or not? for the testing of this system we have to see the spectrum signal of an original speech signal and the spectrum after denoising the noised speech if we get the exact replica or a same spectrum that we are having at the time of an original signal so our system is correct if we don't get the exact replica so our system is not correct. As we have seen in above results here, the spectrum after denoising the speech signal is same as we have the spectrum of an original speech signal it means our results and systems is correct.

\section{REFERENCES}

[1] S. Schilt, L. Thevenaz, M. Nikles, L. Emmenegger, and C. Huglin, "Ammonia monitoring at trace level using photoacoustic spectroscopy inindustrial and environmental applications," Spectrochimica. Acta. Part A: Mol. Biomol. Spectrosc., vol. 60, no. 14, pp. 3259-3268, Dec. 2004.

[2] H. Huszar, A. Pogany, Z. Bozoki, A. Mohacsi, L. Horvath, and G. Szabo, "Ammonia monitoring at ppb level using photoacoustic spectroscopy for environmental application," Sensors and Actuators B: Chemical, vol. 134, no. 2, pp. 10271033, Sep. 2008.

[3] J. Li, X. Gao, W. Li, Z. Cao, L. Deng, W. Zhao, M. Huang, and W. Zhang, "Near-infrared diode laser wavelength modulationbased photoacoustic spectrometer," Spectrochimica. Acta. Part A, vol. 64, no. 2, pp. 338-342, May 2006.

[4] J. P. Besson, S. Schilt, and L. Thevenaz, "Multi-gas sensing based on photoacoustic spectroscopy using tunable laser diodes," Spectrochimica. Acta. Part A, vol. 60, no. 14, pp. 34493456, Dec. 2004.

[5] J. Li, X. Gao, L. Fang, W. Zhang, and H. Cha, "Resonant photoacousti detection of trace gas with DFB diode laser," Opt. Laser Technol., vol. 39, no. 6, pp. 1144-1149, 2007.

[6] M. McCurdy, Y. Bakhirkin, G. Wysocki, R. Lewicki, and F. Tittle, "Recent advances of laser-spectroscopy based techniques for applications in breath analysis", Journal of Breath Research, vol. 1, pp. R1-R12, 2007.

[7] C. Du, J. Zhou, H. Wang, X. Chen, A.Zhu, and J. Zhang, "Determination of soil properties using Fourier transform midinfrared photoacoustic spectroscopy," Vibrational Spectroscopy, to be published.

[8] S. Schilt, A. Vicet, R. Werner, M. Mattiello, L. Thevenaz, A. Salhi, Y. Rouillard, and J. Koeth, "Application of antimonide diode laser in photoacoustic spectroscopy," SpectrochimicaActa Part A, vol. 60, no. 14, pp. 3431-3436, Dec. 2004.

[9] S.Schilt and L. Thevenaz, "Wavelength modulation photoacoustic spectroscopy: Theoretical description and experimental results," Infrared Physics \& Technology, vol. 48, no. 2, pp. 154-162, Jun. 2006.

[10] M. Angelmahr, A. Miklos, and P. Hess, "Wavelength- and amplitudemodulationphotoacoustics: comparison of simulated and measured spectra of higher harmonics," Applied Optics, vol.47, no.15, pp.2806- 2812, May 2008.

[11] J. Besson, S. Schilt, and L. Thevenaz, "Sub-ppb ammonia detection based on photoacoustic spectroscopy," 17th International Conference on Optical Fibre Sensors, Proceedings of SPIE, vol. 5855, pp. 415-418, May. 2005.

[12] google search

[13] wikipedea

[14] Digital Signal processing (theory analysis and design filter design)by B. Somanathan Nair.

[15] Digital signal processing By S.Saliwahanan\& A. Vallavraj. 